\title{
Lack of association between dietary inflammatory index and low impact fractures in the Brazilian population: the Brazilian Osteoporosis Study (BRAZOS)
}

\author{
Melissa Morimoto ${ }^{1 *}$ D, Nitin Shivappa ${ }^{2,3}$, Patricia de Souza Genaro ${ }^{4}$, Lígia Araújo Martini ${ }^{5}$, \\ Natielen Jacques Schuch ${ }^{5}$, James R. Hebert ${ }^{2}$ and Marcelo Medeiros Pinheiro ${ }^{1}$
}

\begin{abstract}
Introduction: Adequate nutrition, including intake of dietary calcium and vitamin $\mathrm{D}$, is important to maintain bone health. Evidence suggests that a deficiency in micronutrients may contribute to bone loss during aging and exert generalized effects on chronic inflammation. Recently, the Dietary Inflammatory Index (DII) was developed to assess the inflammatory potential of individual diets. Our aim was to evaluate the DII in a representative sample and verify its association with low-impact fractures.
\end{abstract}

Methods: Individuals from The Brazilian Osteoporosis Study (BRAZOS) database had their DII calculated. BRAZOS is an important cross-sectional epidemiological study carried out with a representative sample of men and women $\geq 40$ years old. The research was conducted through in-home interviews administered by a trained team. Nutrition Database System for Research (NDSR) software was used to analyze data on the intake of nutrients, which were employed to calculate the DII using Statistical Analysis Software (SAS ${ }^{\circledR}$ ) and Statistical Package for the Social Sciences (SPSS ${ }^{\circledR}$ ) to assess its association with low-impact fractures.

Results: A total of 2269 subjects had their DII score calculated using information from 24-h recall data. Males had lower DII than females ( $D \|=1.12 \pm 1.04$ vs $D \|=1.24 \pm 0.99, p=0.012$ ). Women taking statins had lower $\mathrm{D} \|(\mathrm{D} \|=0.65 \pm 1.14$ vs DII $+1.26 \pm 0.98, p=0.002$ ), indicating a greater potential for diet-related anti-inflammatory effects.

Conclusion: Our findings suggest that women might have a pro-inflammatory diet pattern compared to men. However, we did not find any association between DII scores and low-impact fractures.

Keywords: Diet, Inflammation, Low-impact fractures, Osteoporosis, Dietary inflammatory index

\section{Introduction}

Osteoporosis is a systemic skeletal disease characterized by loss of bone mineral density (BMD), impairment of resistance and bone microarchitecture, and higher risk for low-impact fractures [1]. According to the Brazilian Osteoporosis Study (BRAZOS), about 15.1\% of women and $12.8 \%$ of men reported fragility fractures [2]. More recently, two Brazilian epidemiological studies, SAPOS and SAPORI (Sao Paulo Osteoporosis Study and Sao Paulo

\footnotetext{
* Correspondence: morimotomelissa@gmail.com

${ }^{1}$ Rheumatology Division, Universidade Federal de São Paulo, Unifesp/EPM,

Rua Leandro Dupre, 204, conjunto 74, São Paulo, Brazil

Full list of author information is available at the end of the article
}

Osteoporosis Risk Index), showed that 33\% of postmenopausal women had osteoporosis as diagnosed by Dual-energy X-ray absorptiometry (DXA) measurements, supporting the original findings of BRAZOS $[3,4]$.

Chronic inflammation is associated with a number of chronic non-communicable diseases (CNCDs), including cancer, cardiovascular diseases, obesity, and diabetes mellitus [5]. It is also related to a higher risk of fractures in women, particularly those with higher CRP [6]. In addition, recent evidence indicates a relationship between oxidative stress and osteoporosis, but its role in fractures is still poorly understood $[7,8]$. Therefore, the

(c) The Author(s). 2019 Open Access This article is distributed under the terms of the Creative Commons Attribution 4.0 International License (http://creativecommons.org/licenses/by/4.0/), which permits unrestricted use, distribution, and 
intake of antioxidants could possibly influence BMD in a positive way.

Adequate nutrition, including dietary calcium and vitamin D intake, along with healthy lifestyle changes, are important approaches to minimize bone loss with aging and potential targets for intervention in preventing osteoporosis [9]. Certain micronutrients, including polyphenols, vitamins, polyunsaturated fatty acids (PUFAs), and carotenoids have anti-inflammatory and antioxidant properties [10]. These micronutrients as part of a healthy dietary pattern can help modulate inflammation and oxidative stress and also may be associated with lower CRP levels $[11,12]$. Several studies conducted in different populations have shown that the Mediterranean pattern, comprising grains, fruits, vegetables, olive oil, low-fat dairy products, poultry, and nuts, is associated with lower serum levels of CRP and higher plasma levels of adiponectin, suggesting an anti-inflammatory role [13-15]. In contrast, the Western dietary pattern, characterized by a high intake of red meat, high-fat dairy products, and refined grains, is associated with higher CRP serum levels [16].

The Dietary Inflammatory Index $\left(\mathrm{DII}^{\circ}\right)$ was developed to provide an overall score of the inflammatory potential of individual diets, based on actual food consumption data, in order to predict levels of inflammatory markers [17]. The DII has been validated with inflammatory markers, including associations with CRP $[18,19]$, interleukin-6 [20], and homocysteine [21]. It has also been associated with the glucose intolerance and dyslipidemic components of metabolic syndrome [19], asthma [20], and prostate cancer [22].

Recently, three studies have been conducted to examine the relationship between DII scores and fractures [23-25]. The first one was conducted in a large sample of American women, reported finding that high DII scores, indicating a more inflammatory diet, was associated with increased hip fracture risk. However, this finding was limited only to White women [23]. A case-control study in China, confirmed these findings, in both genders, suggesting that pro-inflammatory diet (with lower DII scores) could be positively associated with higher hip fracture risk [24]. More recently, the Osteoarthritis Initiative study, a longitudinal study with a follow-up of 8 years included 3648 participants, showed that higher DII scores were associated with a higher incidence of fractures, but only in women [25].

We hypothesized that DII would be higher, indicating a more pro-inflammatory diet, in individuals with low-impact fractures. This is the first study to evaluate and describe the inflammatory potential of individuals' diets in a representative sample of the Brazilian adult population.

\section{Material and methods}

\section{Subjects}

Data were included from the BRAZOS study. Briefly, BRAZOS is a cross-sectional population-based study that evaluated age, demographic and anthropometric data, socio-economical aspects, general knowledge about osteoporosis, recurrent falls, medical history, previous fracture, gynecological and reproductive history, familial history of hip fracture after 50 years of age in first-degree relatives, quality of life, current concomitant medication, and comorbidities [2]. Fragility or low-impact fracture was defined as those associated with a fall from standing height or less after age 50 years. Skeletal sites for fragility fractures were axial (ribs, lumbar and thoracic vertebrae) and peripheral bones (forearm, humerus, and femur). Traumatic fractures and those occurring at sites not characteristic of bone fragility (face, skull, tibia, fibula and femoral diaphysis) were excluded from the analysis. Individuals experiencing two or more falls in the previous 12 months were defined as chronic fallers. The presence of cognitive deficiencies (neurological diseases or senile dementia) that could impair the participant's ability to provide informed consent and trustworthy data were excluded from participating in the study. Only one individual $>40$ years old per domicile was permitted to participate. All participants gave written informed consent prior to participation in the study and the research ethics committee of the Universidade Federal de São Paulo/Escola Paulista de Medicina approved with the protocol number 1738/05.

\section{Food intake and dietary inflammatory index}

To compute the DII, we analyzed data from one 24-h recall interview (24HR). Methods of calculating the DII from the food parameters have been described previously [17]. Briefly, the dietary data were first linked to a world database that provides a robust estimate of mean and standard deviation for each food parameter included in the DII. These parameters then became multipliers to express an individual's exposure relative to the "standard global mean" as a z-score. This was achieved by subtracting the "standard global mean" from the amount reported and dividing this value by the standard deviation. To minimize the effect of right-skewing, this value was then converted to a centered proportion score. The centered proportion score for each food parameter and subject was then multiplied by the respective food parameter effect score in order to obtain a food parameter-specific DII score. All of the food parameter-specific DII scores were then summed to create the overall DII score for each study subject.

DII scores were categorized into sex-specific quartiles. The greater the DII score, the more pro-inflammatory the diet; more negative values represent a more anti-inflammatory pattern. Participants reported food and beverages consumed the day before in detail. The 24HR was administered at home and filled out by an interviewer trained by an experienced nutritionist in this 
method. Food data were converted to the respective values of macro and micronutrients using Nutrition Data System for Research version 2005 software (NDSR, University of Minnesota). Energy was adjusted using the residual method described by Willett and Stampfer [26]. Reference values were analyzed considering The Dietary Reference Intakes (DRIs) [27-29] according to age and gender.

In total, 24 of 45 possible food parameters were analyzed to obtain the overall DII score. These included energy intake, carbohydrates, total fat, proteins, cholesterol, saturated fatty acids, monounsaturated fatty acids (MUFAs), PUFAs, fiber, vitamins A, D and E, and minerals such as magnesium, zinc, and selenium.

\section{Anthropometrics}

Body weight $(\mathrm{kg})$ was measured to the nearest $0.1 \mathrm{~kg}$ using a balance beam scale after removal of shoes and heavy outer clothing. Height $(\mathrm{cm})$ was measured after removal of shoes using a stadiometer. Height and body weight were used to calculate body mass index (BMI, $\mathrm{kg} / \mathrm{m}^{2}$ ). Nutritional status was categorized according to WHO criteria [30].

\section{Statistical analysis}

Data are presented as mean and standard deviation (SD) for continuous variables or as frequency and proportion for categorical variables. Mann-Whitney test was used to compare continuous variables for two groups, and the Kruskal-Wallis test was used for three or more groups, for which multiple comparisons were performed with Mann-Whitney test with Bonferroni corrections. The Chi-square test was conducted to determine the relationship between categorical variables, and Spearman correlation coefficients were computed for continuous variables.

For testing effects on fractures, DII was converted to quartiles based on the frequency distribution in the overall population. Race was dichotomized into 'White' and 'non-White'. BMI was categorized into underweight $\left(<18.5 \mathrm{~kg} / \mathrm{m}^{2}\right)$, normal weight $\left(18.5\right.$ to $\left.<25.0 \mathrm{~kg} / \mathrm{m}^{2}\right)$, overweight $\left(25.0\right.$ to $\left.<30.0 \mathrm{~kg} / \mathrm{m}^{2}\right)$, and obese $(\geq 30.0 \mathrm{~kg} /$ $\mathrm{m}^{2}$ ) according to WHO classification [30]. Socioeconomic status (SES) was categorized according to the Brazilian Institute of Geography and Statistics (IBGE) [31] as classes A and B (greater or equal to 15 times the minimum wage), $\mathrm{C}$ (3 to 5 times the minimum wage), and $D$ and $E$ ( 1 to 3 times the minimum wage).

Logistic regression analysis models were designed using DII as the independent factor to predict low-impact fractures. All analysis were performed using SPSS $^{\odot}$ software version 22.0 for Windows (SPSS Inc., Chicago, Illinois). The significance threshold was set at 0.05. The DII scores were calculated using SAS .

\section{Results}

A total of 2269 individuals had their DII score calculated. We excluded data from 151 participants, mostly women, who had insufficient data or unreliable caloric intake (lower than $500 \mathrm{kcal} /$ day or higher than $5.000 \mathrm{kcal} /$ day). Socioeconomic class, demographics, and lifestyle habits characteristics are shown in Tables 1 and 2. There were no significant differences between genders in terms of race, geographic regions, area, and current smoking status. The mean age of participants was $59.7 \pm 13.5$ years and was higher among women $(60.1 \pm 13.7$ years $)$. Most participants were from socio-economic classes D and E $(53.8 \%$ of total), which correspond to incomes of 1 to 3 times minimum wage (lowest level income).

Current and regular alcohol consumption was significantly higher among males (43.1\%) than females (20.6\%), with a mean intake of $4.15 \pm 6.45$ drinks per day (Table $1 ; p<$ 0.001). Smoking was also significantly higher among men. The prevalence of hypertension, dyslipidemia, osteoporosis, rheumatoid arthritis, and gastritis was higher among women; hence, the use of non-steroidal anti-inflammatory drugs (NSAIDs), statins, and supplements such as calcium and vitamins also were higher in women.

Anthropometric data and food intake are shown in Table 2. According to the WHO classification of BMI (Table 2) $20.9 \%$ of females were obese versus $17.9 \%$ of males $(p=0.01)$. Energy intake was higher among male subjects compared to females. However, females had a higher intake of carbohydrates $(p=0.02)$ and trans fat $(p<0.001)$. As a result, DII was also higher among females than males $(p=0.01)$ (Table 3$)$.

\section{Dietary inflammatory index}

As shown in Table 3, mean DII score differed significantly between gender, age groups, region, and socioeconomic classes. Males between 50 and 60 years old had lower DII scores than males aged 41 to 50 years and over 71 years $(p=0.01)$. Subjects from South $(\mathrm{DII}=+1.12)$ and Central (DII $=+1.11)$ regions had lower scores indicating more anti-inflammatory diets compared to those from the Southeast ( $\mathrm{DII}=+1.19)$, North $(\mathrm{DII}=+1.27)$, and Northeast $(\mathrm{DII}=+1.36)$ regions. Males from the Central region had significantly lower scores than those from the South $(p=0.03)$, Northeast $(p=0.04)$ and North $(p=0.01)$. Moreover, females from the South region had lower scores than those from the North ( $p=$ $0.03)$ and Northeast $(p=0.049)$. Socioeconomic classes $\mathrm{D}$ and $\mathrm{E}$ (lowest income) had a higher DII score compared to the other socioeconomic classes (A, B, and C). Males with higher income had lower DII than subjects with lower income.

When DII was converted into quartiles according to gender (Tables 4 and 5), men in quartile 2 were mostly from the Southeast and Central regions $(p=0.006)$. 
Table 1 Demographic and clinical characteristics of the participants - The Brazilian Osteoporosis Study

\begin{tabular}{|c|c|c|c|c|c|c|c|c|c|c|}
\hline \multirow[t]{2}{*}{ Characteristic } & \multicolumn{3}{|c|}{ Total $(n=2269)$} & \multicolumn{3}{|c|}{ Males $(n=684)$} & \multicolumn{3}{|c|}{ Females $(n=1585)$} & \multirow[t]{2}{*}{$P$ value } \\
\hline & Mean or $n$ & SD & $\%$ & Mean or $n$ & SD & $\%$ & Mean or $n$ & SD & $\%$ & \\
\hline Race & & & & & & & & & & 0.16 \\
\hline White & 1072 & - & 47.2 & 308 & - & 45.0 & 764 & - & 48.2 & \\
\hline Non-white & 1197 & - & 52.8 & 376 & - & 55.0 & 821 & - & 51.8 & \\
\hline Age group & & & & & & & & & & $0.03^{\mathrm{a}}$ \\
\hline $40-50$ years & 785 & - & 34.6 & 249 & - & 36.4 & 536 & - & 33.8 & \\
\hline $51-60$ years & 443 & - & 19.5 & 152 & - & 22.2 & 291 & - & 18.4 & \\
\hline $61-70$ years & 418 & - & 18.4 & 114 & - & 16.7 & 304 & - & 19.2 & \\
\hline$\geq 71$ years & 623 & - & 27.5 & 169 & - & 24.7 & 454 & - & 28.6 & \\
\hline Social class ${ }^{d}$ & & & & & & & & & & $0.03^{b}$ \\
\hline$A B$ & 314 & - & 13.8 & 109 & - & 15.9 & 205 & - & 12.9 & \\
\hline C & 735 & - & 32.4 & 234 & - & 34.2 & 501 & - & 31.6 & \\
\hline DE & 1220 & - & 53.8 & 341 & - & 49.9 & 879 & - & 55.5 & \\
\hline Geographic region & & & & & & & & & & 0.88 \\
\hline North & 297 & - & 13.1 & 94 & - & 13.7 & 203 & - & 12.8 & \\
\hline Northeast & 475 & - & 20.9 & 136 & - & 19.9 & 339 & - & 21.4 & \\
\hline Central-West & 330 & - & 14.5 & 102 & - & 14.9 & 228 & - & 14.4 & \\
\hline South & 372 & - & 16.4 & 116 & - & 17.0 & 256 & - & 16.2 & \\
\hline Southeast & 705 & - & 35.0 & 236 & - & 34.5 & 559 & - & 35.3 & \\
\hline Area & & & & & & & & & & 0.68 \\
\hline Metropolitan & 1103 & - & 48.6 & 328 & - & 48.0 & 775 & - & 48.9 & \\
\hline Interior & 1166 & - & 51.4 & 356 & - & 52.0 & 810 & - & 51.1 & \\
\hline Marital status & & & & & & & & & & $<0.001^{c}$ \\
\hline Single & 245 & - & 10.8 & 70 & - & 10.2 & 175 & - & 11 & \\
\hline Married & 1240 & - & 54.6 & 478 & - & 69.9 & 762 & - & 48.1 & \\
\hline Widowed & 585 & - & 25.8 & 82 & - & 12.0 & 503 & - & 31.7 & \\
\hline Divorced & 199 & - & 8.7 & 54 & - & 7.9 & 145 & - & 9.2 & \\
\hline Alcohol consumption & & & & & & & & & & $<0.001$ \\
\hline Yes & 621 & - & 27.3 & 295 & - & 43.1 & 326 & - & 20.6 & \\
\hline No & 1648 & - & 72.6 & 389 & - & 56.9 & 1259 & - & 79.4 & \\
\hline Mean & 2.22 & 4.6 & & 4.15 & 6.45 & & 1.38 & 3.17 & 3.17 & $<0.001$ \\
\hline Smoking status & & & & & & & & & & $<0.001$ \\
\hline Current smoker & 438 & - & 19.3 & 166 & - & 24.3 & 272 & - & 17.2 & \\
\hline Past & 723 & - & 31.9 & 269 & - & 39.4 & 453 & - & 28.6 & \\
\hline Never & 1108 & - & 48.8 & 248 & - & 36.3 & 860 & - & 54.3 & \\
\hline Cigarettes/day & 15.12 & 15.50 & - & 17.64 & 14.26 & - & 13.6 & 16.07 & - & $<0.001$ \\
\hline Age started (years) & 18.05 & 11.50 & - & 15.64 & 6.87 & - & 19.5 & 13.40 & - & $<0.001$ \\
\hline Age quit (years) & 42.75 & 16.50 & - & 41.38 & 14.98 & - & 43.5730 & 45 & - & 0.23 \\
\hline Low impact Fractures & & & & & & & & & & 0.006 \\
\hline Yes & 205 & - & 9.0 & 79 & - & 11.5 & 126 & - & 7.9 & \\
\hline No & 2064 & - & 91.0 & 605 & - & 88.5 & 1459 & - & 92.1 & \\
\hline Diabetes Mellitus & 236 & - & 7.4 & 64 & - & 9.4 & 172 & - & 10.9 & 0.28 \\
\hline Hypertension & 790 & - & 24.6 & 197 & - & 28.8 & 593 & - & 37.4 & $<0.001$ \\
\hline Dyslipidemia & 175 & - & 5.5 & 30 & - & 4.4 & 145 & - & 9.1 & $<0.001$ \\
\hline
\end{tabular}


Table 1 Demographic and clinical characteristics of the participants - The Brazilian Osteoporosis Study (Continued)

\begin{tabular}{|c|c|c|c|c|c|c|c|c|c|c|}
\hline \multirow[t]{2}{*}{ Characteristic } & \multicolumn{3}{|c|}{ Total $(n=2269)$} & \multicolumn{3}{|c|}{ Males $(n=684)$} & \multicolumn{3}{|c|}{ Females $(n=1585)$} & \multirow[t]{2}{*}{$P$ value } \\
\hline & Mean or $n$ & SD & $\%$ & Mean or $\mathrm{n}$ & SD & $\%$ & Mean or $n$ & SD & $\%$ & \\
\hline Osteoporosis & 214 & - & 6.7 & 21 & - & 3.1 & 193 & - & 12.2 & $<0.001$ \\
\hline Rheumatoid Arthritis & 259 & - & 8.1 & 34 & - & 5.0 & 225 & - & 14.2 & $<0.001$ \\
\hline Cancer & 31 & - & 1.0 & 10 & - & 1.5 & 21 & - & 1.3 & 0.8 \\
\hline Gastritis & 313 & - & 13.8 & 71 & - & 10.4 & 242 & - & 15.3 & 0.002 \\
\hline \multicolumn{11}{|l|}{ Drug therapy } \\
\hline NSAIDs ${ }^{e}$ & 85 & - & 3.7 & 13 & - & 1.9 & 72 & - & 4.5 & 0.002 \\
\hline Statins & 35 & - & 1.5 & 5 & - & 0.7 & 30 & - & 1.9 & 0.04 \\
\hline Corticoids & 94 & - & 4.1 & 26 & - & 3.8 & 68 & - & 4.3 & 0.81 \\
\hline $\mathrm{HRT}^{f}$ & - & - & - & - & - & - & 97 & - & 6.1 & \\
\hline
\end{tabular}

SD Standard deviation

${ }^{\mathrm{a} D i f f e r e n c e ~ w a s ~ o b s e r v e d ~ o n ~} 51-60$ age group $(p=0.033)$, using Bonferroni correction

${ }^{b} \mathrm{D}$ and $\mathrm{E}$ classes had more frequency than the other social classes $(p=0.014)$

${ }^{c}$ Married and widowed were majority in this study $(p<0.001)$

${ }^{\mathrm{d}}$ Classes $\mathrm{A}$ and $\mathrm{B}$ (earning 15 times more than minimum wages), $\mathrm{C}$ ( 3 to 5 times minimum wage), and $\mathrm{D}$ and $\mathrm{E}$ ( 1 to 3 times minimum wage)

${ }^{\mathrm{N}}$ NSAIDs: nonsteroidal anti-inflammatory drugs

f $H R T$ hormone replacement therapy

Table 2 Anthropometric data, food consumption and lifestyle characteristics of the participants

\begin{tabular}{|c|c|c|c|c|c|c|c|c|c|c|}
\hline \multirow[t]{2}{*}{ Characteristic } & \multicolumn{3}{|c|}{ Total $(n=2269)$} & \multicolumn{3}{|c|}{ Males $(n=684)$} & \multicolumn{3}{|c|}{ Females $(n=1585)$} & \multirow[t]{2}{*}{$P$ value } \\
\hline & Mean or $n$ & SD & $\%$ & Mean or $n$ & SD & $\%$ & Mean or $n$ & SD & $\%$ & \\
\hline Weight (kg) & 67.30 & 14.50 & - & 73.40 & 14.69 & - & 64.64 & 13.67 & - & $<0.001$ \\
\hline Height (m) & 1.59 & 0.10 & - & 1.66 & 0.07 & - & 1.56 & 0.09 & - & $<0.001$ \\
\hline BMI $\left(\mathrm{kg} / \mathrm{m}^{2}\right)$ & & & & & & & & & & 0.01 \\
\hline Underweight (<18.5) & 69 & - & 3.0 & 18 & - & 2.7 & 51 & - & 3.3 & \\
\hline Normal weight (18.5-25) & 889 & - & 39.2 & 251 & - & 37.4 & 638 & - & 41.1 & \\
\hline Overweight (25-30) & 821 & - & 36.2 & 282 & - & 42.0 & 539 & - & 34.7 & \\
\hline Obesity $(\geq 30)$ & 444 & - & 19.5 & 120 & - & 17.9 & 324 & - & 20.9 & \\
\hline Energy intake (kcal/d) & 1335 & 578 & - & 1590 & 698 & - & 1225 & 484 & - & $<0.001$ \\
\hline Carbohydrates (g) & 167.9 & 39.3 & - & 165.1 & 47.6 & - & 169.2 & 35.0 & - & 0.02 \\
\hline Proteins (g) & 39.3 & 22.5 & - & 40.0 & 26.4 & - & 39.1 & 20.7 & - & 0.9 \\
\hline Lipids (g) & 44.2 & 13.6 & - & 43.8 & 15.7 & - & 44.5 & 12.6 & - & 0.28 \\
\hline Trans fat (g) & 2.4 & 2.1 & - & 2.1 & 2.1 & - & 2.6 & 2.1 & - & $<0.001$ \\
\hline Saturated fat (g) & 13.7 & 5.9 & - & 13.5 & 6.8 & - & 13.9 & 5.5 & - & 0.06 \\
\hline Calcium & & & & & & & & & & $<0.001$ \\
\hline Yes & 246 & - & 10.8 & 47 & - & 6.9 & 199 & - & 12.6 & \\
\hline No & 2006 & - & 88.4 & 632 & - & 92.4 & 1374 & - & 86.7 & \\
\hline Calcium + Vitamin D & 21 & - & 0.9 & 3 & - & 0.4 & 18 & - & 1.1 & 0.11 \\
\hline Vitamins & 12 & - & 0.5 & 4 & - & 0.6 & 8 & - & 0.5 & 0.81 \\
\hline \multicolumn{11}{|l|}{ Physical activity* } \\
\hline PEL & 1.58 & 0.76 & - & 1.68 & 0.80 & - & 1.54 & 0.74 & - & $<0.001$ \\
\hline $\mathrm{AFO}$ & 2.38 & 0.62 & - & 2.52 & 0.64 & - & 2.32 & 0.61 & - & $<0.001$ \\
\hline LLA & 1.87 & 0.67 & - & 2.06 & 0.75 & - & 1.79 & 0.62 & - & $<0.001$ \\
\hline TS & 5.84 & 1.69 & - & 6.27 & 1.77 & - & 5.65 & 1.62 & & $<0.001$ \\
\hline
\end{tabular}


Table 3 Dietary Inflammatory Index according to the characteristics of the participants

\begin{tabular}{|c|c|c|c|c|c|c|c|c|c|}
\hline \multirow[t]{3}{*}{ Characteristics } & \multicolumn{9}{|c|}{$\underline{D} \|(n=2269)$} \\
\hline & \multicolumn{3}{|c|}{ Total $(n=2269)$} & \multicolumn{3}{|c|}{ Males $(n=684)$} & \multicolumn{3}{|c|}{ Females $(n=1585)$} \\
\hline & Mean & SD & $P$ value & Mean & SD & $P$ value & Mean & SD & $P$ value \\
\hline Gender & & & 0.01 & & & - & & & - \\
\hline Males & 1.12 & 1.04 & & - & & & - & & \\
\hline Females & 1.24 & 0.99 & & - & & & - & & \\
\hline Age group & & & 0.006 & & & 0.009 & & & 0.34 \\
\hline $40-50$ years & 1.25 & 0.98 & & 1.17 & 1.00 & & 1.29 & 0.96 & \\
\hline $51-60$ years & 1.07 & 1.06 & & 0.93 & 1.03 & & 1.14 & 1.07 & \\
\hline $61-70$ years & 1.19 & 0.98 & & 1.08 & 1.05 & & 1.23 & 0.96 & \\
\hline$\geq 71$ years & 1.26 & 1.01 & & 1.24 & 1.08 & & 1.28 & 0.98 & \\
\hline BMI $\left(\mathrm{kg} / \mathrm{m}^{2}\right)$ & & & 0.45 & & & 0.74 & & & 0.4 \\
\hline Underweight $(<18.5)$ & 1.17 & 1.03 & & 0.87 & 1.37 & & 1.32 & 0.81 & \\
\hline Normal weight (18.5-25) & 1.17 & 1.01 & & 1.12 & 1.05 & & 1.19 & 1.00 & \\
\hline Overweight (25-30) & 1.25 & 0.99 & & 1.17 & 1.04 & & 1.30 & 0.96 & \\
\hline Obesity ( $\geq 30)$ & 1.20 & 1.00 & & 1.12 & 0.95 & & 1.24 & 1.01 & \\
\hline Race & & & 0.08 & & & 0.52 & & & 0.08 \\
\hline White & 1.17 & 0.97 & & 1.10 & 0.99 & & 1.20 & 0.96 & \\
\hline Non-white & 1.24 & 1.03 & & 1.13 & 1.08 & & 1.28 & 1.01 & \\
\hline Geographic region & & & 0.001 & & & 0.006 & & & 0.01 \\
\hline South & 1.12 & 1.03 & & 1.16 & 1.12 & & 1.10 & 0.99 & \\
\hline Southeast & 1.19 & 0.96 & & 1.11 & 1.05 & & 1.23 & 0.92 & \\
\hline Central-West & 1.11 & 0.96 & & 0.84 & 1.00 & & 1.23 & 0.92 & \\
\hline North & 1.27 & 1.10 & & 1.29 & 0.95 & & 1.40 & 0.97 & \\
\hline Northeast & 1.36 & 0.96 & & 1.19 & 1.02 & & 1.30 & 1.12 & \\
\hline Area & & & 0.72 & & & 0.23 & & & 0.2 \\
\hline Metropolitan & 1.17 & 1.03 & & 1.07 & 1.03 & & 1.21 & 1.02 & \\
\hline Interior & 1.24 & 0.98 & & 1.16 & 1.05 & & 1.28 & 0.95 & \\
\hline Social class ${ }^{a}$ & & & 0.001 & & & 0.005 & & & 0.02 \\
\hline$A B$ & 1.06 & 1.00 & & 0.81 & 1.13 & & 1.20 & 0.89 & \\
\hline C & 1.18 & 1.00 & & 1.17 & 1.00 & & 1.18 & 1.00 & \\
\hline $\mathrm{DE}$ & 1.26 & 1.00 & & 1.18 & 1.03 & & 1.29 & 0.99 & \\
\hline Alcohol consumption & & & 0.81 & & & 0.2 & & & 0.12 \\
\hline Yes & 1.20 & 1.01 & & 1.08 & 1.01 & & 1.31 & 0.99 & \\
\hline No & 1.21 & 1.00 & & 1.15 & 1.06 & & 1.23 & 0.98 & \\
\hline Smoking status & & & 0.89 & & & 0.86 & & & 0.94 \\
\hline Current smoker & 1.22 & 0.96 & & 1.12 & 1.03 & & 1.28 & 0.91 & \\
\hline Past & 1.19 & 1.01 & & 1.09 & 1.05 & & 1.25 & 0.99 & \\
\hline Never & 1.21 & 1.01 & & 1.15 & 1.05 & & 1.23 & 1.00 & \\
\hline Marital status & & & 0.1 & & & 0.48 & & & 0.25 \\
\hline Single & 1.26 & 1.03 & & 1.12 & 1.18 & & 1.31 & 0.96 & \\
\hline Married & 1.17 & 1.02 & & 1.12 & 1.01 & & 1.21 & 1.02 & \\
\hline Widowed & 1.29 & 0.95 & & 1.26 & 1.05 & & 1.29 & 0.94 & \\
\hline Divorced & 1.13 & 1.15 & & 1.16 & 1.29 & & 1.11 & 1.10 & \\
\hline
\end{tabular}

SD Standard deviation, $B M I$ Body mass index

${ }^{a}$ Classes $A$ and $B$ (earning 15 times more than minimum wage), $C$ ( 3 to 5 times minimum wage), and $D$ and $E$ ( 1 to 3 times minimum wage) 
Table 4 Characteristics of the participants according to DIl quartile for Brazilian men

\begin{tabular}{|c|c|c|c|c|c|c|c|c|c|}
\hline \multirow[t]{2}{*}{ Characteristics } & \multicolumn{2}{|l|}{$\begin{array}{l}\text { Quartile } 1 \\
(\leq+0.49)\end{array}$} & \multicolumn{2}{|c|}{$\begin{array}{l}\text { Quartile } 2 \\
(+0.49 a+1.29)\end{array}$} & \multicolumn{2}{|c|}{$\begin{array}{l}\text { Quartile } 3 \\
(+1.29 a+1.89)\end{array}$} & \multicolumn{2}{|l|}{$\begin{array}{l}\text { Quartile } 4 \\
(>1.89)\end{array}$} & \multirow[t]{2}{*}{$P$ value } \\
\hline & Mean or $n$ & SD or $\%$ & Mean or $n$ & SD or $\%$ & Mean or $n$ & SD or $\%$ & Mean or $n$ & SD or $\%$ & \\
\hline Age (years) & 57.8 & 12.3 & 57.5 & 12.0 & 58.1 & 12.9 & 59.8 & 14.2 & 0.6 \\
\hline Weight (kg) & 74.2 & 14.7 & 74.4 & 15.3 & 73.0 & 13.6 & 72.0 & 15.2 & 0.3 \\
\hline BMI $\left(\mathrm{kg} / \mathrm{m}^{2}\right)$ & 26.5 & 4.8 & 26.4 & 4.7 & 26.3 & 4.3 & 26.0 & 4.6 & 0.86 \\
\hline \multicolumn{10}{|l|}{ Alcohol } \\
\hline Yes & 76 & 45.0 & 80 & 46.8 & 74 & 43.8 & 65 & 37.4 & 0.31 \\
\hline No & 93 & 55.0 & 91 & 53.2 & 95 & 56.2 & 109 & 62.6 & \\
\hline Mean consumption & 7.3 & 15.5 & 11.2 & 22.3 & 10.3 & 20.3 & 6.3 & 10.8 & 0.35 \\
\hline Geographic region & & & & & & & & & 0.01 \\
\hline South & 27 & 16.0 & 24 & 14.0 & 25 & 14.8 & 39 & 22.4 & \\
\hline Southeast & 54 & 32.0 & 67 & 39.2 & 62 & 36.7 & 53 & 30.5 & \\
\hline Central-West & 31 & 18.3 & 37 & 21.6 & 18 & 10.7 & 16 & 9.2 & \\
\hline North & 22 & 13.0 & 16 & 9.4 & 27 & 16.0 & 29 & 16.7 & \\
\hline Northeast & 35 & 20.7 & 27 & 15.8 & 37 & 21.9 & 37 & 21.3 & \\
\hline Marital status & & & & & & & & & 0.46 \\
\hline Single & 18 & 10.7 & 14 & 8.2 & 15 & 8.9 & 23 & 13.2 & \\
\hline Married & 119 & 70.4 & 127 & 74.3 & 118 & 69.8 & 114 & 65.5 & \\
\hline Widowed & 17 & 10.1 & 18 & 10.5 & 22 & 13.0 & 24 & 13.8 & \\
\hline Divorced/separated & 7 & 4.1 & 5 & 2.9 & 6 & 3.6 & 11 & 6.3 & \\
\hline Low impact fractures & & & & & & & & & 0.14 \\
\hline Yes & 18 & 10.6 & 19 & 11.1 & 14 & 8.3 & 28 & 16.1 & \\
\hline No & 152 & 89.4 & 152 & 88.9 & 155 & 91.7 & 146 & 83.9 & \\
\hline NSAIDs ${ }^{a}$ & 2 & 1.2 & 5 & 2.9 & 4 & 2.4 & 13 & 1.9 & 0.54 \\
\hline Statins & - & - & 3 & 1.8 & - & - & 2 & 1.1 & 0.15 \\
\hline Hypertension & 48 & 28.4 & 49 & 28.7 & 46 & 27.2 & 54 & 31.0 & 0.89 \\
\hline Diabetes mellitus & 19 & 11.2 & 18 & 10.5 & 11 & 6.5 & 16 & 9.2 & 0.46 \\
\hline Osteoporosis & 8 & 4.7 & 4 & 2.3 & 4 & 2.4 & 5 & 2.9 & 0.54 \\
\hline Dyslipidemia & 4 & 2.4 & 10 & 5.8 & 9 & 5.3 & 7 & 4.0 & 0.4 \\
\hline
\end{tabular}

$S D$ Standard deviation, $B M I$ Body mass index

${ }^{a}$ NSAIDs Nonsteroidal anti-inflammatory drugs

Nonetheless, for both genders, those with pro-inflammatory potential (quartile 4) were from the South, North, and Northeast $(p=0.006)$. Women taking statins had significantly lower DII scores $(0.65 \pm 1.14$ vs $1.26 \pm 0.98 ; p=$ $0.002)$. Additionally, women on hormone replacement therapy (HRT) tended to have lower DII $(p=0.06)$. However, when analyzed by quartiles, most of the women on HRT were from quartiles 1 and $2(p=0.03)$ (Table 5).

\section{Fractures}

Overall, 9\% reported low-impact fractures $(11.5 \%$ of men and $7.9 \%$ of women). We did not find any association between low-impact fractures and DII scores. Logistic regression with the DII as an independent variable and fractures as a dependent variable indicated that for every unit increase of the DII score, the chance of having a low-impact fracture was 1.15 times higher $(p=0.08)$. When adjusted for DM and osteoporosis, we found that for every unit increase of the DII score, the chance of having a low-impact fracture was 1.18 times higher $(p=0.08)$ (data shown only in text). However, these results did not reach statistical significance in either analysis (Table 6).

\section{Discussion}

To the best of our knowledge, this is the first population-based study evaluating the association between DII and fragility fractures in a nationally representative sample. Although females had higher DII scores, indicating pro-inflammatory diets compared to males, we did not find any association with low-impact fractures after multiple adjustments. In Iran, postmenopausal women with 
Table 5 Characteristics of the participants according to Dll quartile for Brazilian women

\begin{tabular}{|c|c|c|c|c|c|c|c|c|c|}
\hline \multirow[t]{2}{*}{ Characteristics } & \multicolumn{2}{|c|}{ Quartile $1(\leq+0.69)$} & \multicolumn{2}{|c|}{ Quartile $2(+0.69 a+1.39)$} & \multicolumn{2}{|c|}{ Quartile 3 (+ $1.39 a+1.93)$} & \multicolumn{2}{|c|}{ Quartile 4 (> 1.93) } & \multirow[t]{2}{*}{$P$ value } \\
\hline & Mean or $n$ & SD or $\%$ & Mean or $n$ & SD or $\%$ & Mean or $n$ & SD or $\%$ & Mean or $n$ & SD or $\%$ & \\
\hline Age (years) & 60.7 & 13.6 & 59.4 & 13.8 & 60.4 & 14.0 & 60.4 & 13.7 & 0.44 \\
\hline Weight (kg) & 64.8 & 14.3 & 64.7 & 13.3 & 64.0 & 13.2 & 65.1 & 13.9 & 0.72 \\
\hline BMI $\left(\mathrm{kg} / \mathrm{m}^{2}\right)$ & 26.2 & 5.6 & 26.2 & 5.2 & 26.2 & 4.9 & 26.6 & 5.3 & 0.43 \\
\hline Alcohol & & & & & & & & & 0.35 \\
\hline Yes & 76 & 19.2 & 73 & 18.3 & 88 & 22.3 & 89 & 22.5 & \\
\hline No & 320 & 80.8 & 325 & 81.7 & 307 & 77.7 & 307 & 77.5 & \\
\hline Mean consumption & 5.9 & 17.2 & 7.5 & 20.5 & 5.8 & 14.8 & 3.7 & 7.9 & 0.16 \\
\hline Geographic region & & & & & & & & & 0.006 \\
\hline South & 73 & 18.4 & 68 & 17.1 & 63 & 15.9 & 52 & 13.1 & \\
\hline Southeast & 134 & 33.8 & 149 & 37.4 & 160 & 40.5 & 116 & 29.3 & \\
\hline Central-West & 63 & 15.9 & 58 & 14.6 & 50 & 12.7 & 57 & 14.4 & \\
\hline North & 41 & 10.4 & 49 & 12.3 & 50 & 12.7 & 108 & 27.3 & \\
\hline Northeast & 85 & 21.5 & 74 & 18.6 & 72 & 18.2 & 63 & 15.9 & \\
\hline Marital status & & & & & & & & & 0.35 \\
\hline Single & 41 & 10.4 & 39 & 9.8 & 45 & 11.4 & 50 & 12.6 & \\
\hline Married & 203 & 51.3 & 190 & 47.7 & 190 & 48.1 & 179 & 45.2 & \\
\hline Widowed & 115 & 29.0 & 132 & 33.2 & 126 & 31.9 & 130 & 32.8 & \\
\hline Divorced/separated & 26 & 6.6 & 16 & 4.0 & 13 & 3.3 & 19 & 4.8 & \\
\hline Low impact fractures & & & & & & & & & 0.15 \\
\hline Yes & 37 & 9.3 & 35 & 8.8 & 21 & 5.3 & 33 & 8.3 & \\
\hline No & 359 & 90.7 & 363 & 91.2 & 374 & 94.7 & 363 & 91.7 & \\
\hline $\mathrm{NSAIDs}^{\mathrm{a}}$ & 20 & 5.1 & 22 & 5.5 & 11 & 2.8 & 19 & 4.8 & 0.26 \\
\hline Statins & 16 & 4.0 & 6 & 1.5 & 3 & 0.8 & 5 & 1.3 & 0.003 \\
\hline Hypertension & 26 & 17.2 & 35 & 22.6 & 20 & 12.7 & 16 & 11.3 & 0.03 \\
\hline Diabetes mellitus & 155 & 39.1 & 155 & 38.9 & 149 & 37.7 & 134 & 33.8 & 0.38 \\
\hline Osteoporosis & 41 & 10.4 & 53 & 13.3 & 47 & 11.9 & 31 & 7.8 & 0.08 \\
\hline Dyslipidemia & 53 & 13.4 & 48 & 12.1 & 47 & 11.9 & 45 & 11.4 & 0.84 \\
\hline
\end{tabular}

$S D$ Standard deviation, $B M I$ Body mass index

${ }^{a}$ NSAIDs Nonsteroidal anti-inflammatory drugs

higher DII scores were more likely to have lower spine BMD measurements [32]. Our findings are similar to the Osteoarthritis Initiative that did not find a statistically significant association between higher DII scores and fractures in men, although it did find higher DII scores in women with fractures [25].

The DII was associated with higher serum levels of CPR in the Seasonal Variation of Blood Cholesterol Study (SEASONS), and CRP of more than $3.0 \mathrm{mg} / \mathrm{L}$ was predicted for each one-unit increase in the DII (OR = 1.08; $\mathrm{IC}=1.01$ to 1.16 ) based on either $24 \mathrm{HR}$ or 7 -day recalls [18]. When associated with metabolic syndrome among police officers, DII quartiles 2 to 4 (more pro-inflammatory diets) were more likely to exceed a threshold of $3.0 \mathrm{mg} / \mathrm{L}$ for CRP than quartile 1 [19]. In Australia, diet consumed by subjects with asthma was more pro-inflammatory than in healthy controls, and higher DII was associated with impaired lung function [20]. Higher DII scores, especially in males, also were associated with increased risk of colorectal cancer [23].

Interestingly, the profile of women taking statins in our study was associated with lower DII scores. Although these lower levels can be indicating lower systemic inflammation, the cross-sectional design did not allow us to demonstrate a temporal relationship among these variables [33]. Moreover, low scores may be related to food intake advice given by health professionals and more prospective studies are necessary to establish an effect and causal relationship.

Contrary to our hypothesis, higher BMI was not associated with a more pro-inflammatory diet, emphasizing that some nutrients could have a more positive role in the DII than simply that attributed to total energy consumption and BMI. Considering that nutrients or foods 
Table 6 Dietary Inflammatory Index, according to gender and concomitant diseases

\begin{tabular}{|c|c|c|c|c|c|c|c|c|c|}
\hline \multirow[t]{2}{*}{ Characteristics } & \multicolumn{3}{|c|}{ Total $(n=2269)$} & \multicolumn{3}{|c|}{ Males $(n=684)$} & \multicolumn{3}{|c|}{ Females $(n=1585)$} \\
\hline & Mean & SD & $P$ value & Mean & SD & $P$ value & Mean & SD & $P$ value \\
\hline \multicolumn{10}{|c|}{ Low-impact fractures } \\
\hline Yes & 1.22 & 1.06 & 0.71 & 1.28 & 1.13 & 0.09 & 1.18 & 1.02 & 0.48 \\
\hline No & 1.21 & 1.00 & & 1.10 & 1.03 & & 1.25 & 0.99 & \\
\hline \multicolumn{10}{|l|}{ Diseases } \\
\hline \multicolumn{10}{|l|}{ Diabetes Mellitus } \\
\hline Yes & 1.12 & 0.98 & 0.07 & 0.95 & 1.10 & 0.14 & 1.18 & 0.93 & 0.19 \\
\hline No & 1.22 & 1.01 & & 1.14 & 1.04 & & 1.26 & 1.00 & \\
\hline \multicolumn{10}{|l|}{ Hypertension } \\
\hline Yes & 1.18 & 1.00 & 0.22 & 1.12 & 1.02 & 0.98 & 1.19 & 1.00 & 0.08 \\
\hline No & 1.23 & 1.01 & & 1.12 & 1.06 & & 1.28 & 0.99 & \\
\hline \multicolumn{10}{|l|}{ Dyslipidemia } \\
\hline Yes & 1.20 & 0.97 & 0.93 & 1.29 & 0.89 & 0.57 & 1.18 & 0.99 & 0.55 \\
\hline No & 1.21 & 1.01 & & 1.12 & 1.05 & & 1.26 & 0.99 & \\
\hline \multicolumn{10}{|l|}{ Osteoporosis } \\
\hline Yes & 1.18 & 1.00 & 0.37 & 0.96 & 1.11 & 0.37 & 1.20 & 0.99 & 0.29 \\
\hline No & 1.21 & 1.01 & & 1.13 & 1.04 & & 1.26 & 0.99 & \\
\hline \multicolumn{10}{|c|}{ Rheumatoid arthritis } \\
\hline Yes & 1.18 & 1.01 & 0.42 & 1.21 & 0.75 & 0.92 & 1.18 & 1.05 & 0.21 \\
\hline No & 1.21 & 1.01 & & 1.12 & 1.06 & & 1.26 & 0.98 & \\
\hline \multicolumn{10}{|l|}{ Cancer } \\
\hline Yes & 1.21 & 1.04 & 0.99 & 1.17 & 0.89 & 0.99 & 1.23 & 1.12 & 0.99 \\
\hline No & 1.21 & 1.01 & & 1.12 & 1.05 & & 1.25 & 0.99 & \\
\hline \multicolumn{10}{|l|}{ Gastritis } \\
\hline Yes & 1.21 & 1.01 & 0.83 & 1.17 & 1.03 & 0.67 & 1.22 & 1.00 & 0.5 \\
\hline No & 1.21 & 1.01 & & 1.12 & 1.05 & & 1.25 & 0.99 & \\
\hline \multicolumn{10}{|l|}{ Asthma } \\
\hline Yes & 1.16 & 1.02 & 0.78 & 1.32 & 0.92 & 0.17 & 1.08 & 1.06 & 0.2 \\
\hline No & 1.21 & 1.01 & & 1.11 & 1.05 & & 1.26 & 0.99 & \\
\hline \multicolumn{10}{|l|}{ Drug therapy } \\
\hline \multicolumn{10}{|l|}{ NSAIDs ${ }^{a}$} \\
\hline Yes & 1.23 & 1.04 & 0.66 & 1.20 & 0.67 & 0.91 & 1.24 & 1.09 & 0.54 \\
\hline No & 1.21 & 1.01 & & 1.12 & 1.05 & & 1.25 & 0.99 & \\
\hline \multicolumn{10}{|l|}{ Statins } \\
\hline Yes & 0.75 & 1.11 & 0.009 & 1.32 & 0.74 & 0.76 & 0.65 & 1.14 & 0.002 \\
\hline No & 1.22 & 1.01 & & 1.12 & 1.05 & & 1.26 & 0.98 & \\
\hline Corticoids & & & 0.5 & & & 0.06 & & & 0.95 \\
\hline Yes & 1.10 & 1.04 & & 0.70 & 1.05 & & 1.26 & 1.00 & \\
\hline No & 1.22 & 1.01 & & 1.14 & 1.03 & & 1.25 & 0.99 & \\
\hline Premenopausal & - & & & - & & & 1.26 & 0.98 & 0.49 \\
\hline Menopausal & - & & & - & & & 1.21 & 0.98 & 0.25 \\
\hline$H R T^{b}$ & - & & & - & & & & & 0.06 \\
\hline
\end{tabular}


Table 6 Dietary Inflammatory Index, according to gender and concomitant diseases (Continued)

\begin{tabular}{|c|c|c|c|c|c|c|c|c|c|}
\hline \multirow[t]{2}{*}{ Characteristics } & \multicolumn{3}{|c|}{ Total $(n=2269)$} & \multicolumn{3}{|c|}{ Males $(n=684)$} & \multicolumn{3}{|c|}{ Females $(n=1585)$} \\
\hline & Mean & SD & $P$ value & Mean & SD & $P$ value & Mean & SD & $P$ value \\
\hline Yes & - & & & - & & & 1.02 & 1.09 & \\
\hline No & - & & & - & & & 1.25 & 0.95 & \\
\hline Calcium & & & 0.64 & & & 0.87 & & & 0.66 \\
\hline Yes & 1.39 & 0.98 & & 1.17 & 0.90 & & 1.32 & 1.00 & \\
\hline No & 1.21 & 0.98 & & 1.12 & 1.05 & & 1.25 & 0.99 & \\
\hline
\end{tabular}

SD Standard deviation

${ }^{a}$ NSAIDs Nonsteroidal anti-inflammatory drugs

${ }^{\mathrm{b}} H R T$ Hormone replacement therapy

are rarely eaten in isolation, dietary patterns may have synergistic or antagonistic biochemical interactions among nutrients as well as different food sources of the same nutrient. Instead of looking at individual nutrients or foods, pattern analysis examines the effects of the overall diet. Conceptually, dietary patterns represent a broader picture of food and nutrient consumption and may be more predictive of higher risk of diseases than the individual intake of foods or nutrients [34, 35].

Our previously published studies have shown some relationships between nutrient intake and osteoporotic fractures such as consumption of antioxidants [36], and caffeine [37], as well as clinical risks factors for fractures [2], recurrent falls [38], and quality of life [39]. Consumption of solid fats and added sugars (SoFAS) in Brazil corresponds to $52 \%$ of daily intake, and they are provided from food with empty calories, especially in women and teenagers [40]. This outcome also was observed in our study. Women from BRAZOS had a higher consumption of carbohydrates and trans fat compared to men, resulting in higher DII scores.

A possible explanation for the association between DII scores and lower income could be explained by the inadequate consumption of fruits and vegetables, which tend to be expensive. According to Sichieri and colleagues [41], the traditional pattern is mostly determined by socioeconomic conditions, and apart from that, is a protection for overweight and obesity. However, the antioxidant intake was low in the adult Brazilian population [36], regardless of social class, economic status, race, or region of the country.

The latest Household Budget Survey report describing food intake of the Brazilian population also showed a positive association between consumption of vegetables and fruits and socio-economic classes and a negative association with manioc flour [42]. Fruits, vegetables, skim milk, and dairy intake increased proportionately to higher income. When categorized by the 5 regions of Brazil, the Central region, which had the lowest DII scores (most anti-inflammatory) in our study, had a higher consumption of rice, beans, red meat, and grains.
The South region, with the second lowest DII scores in our study, had a higher consumption of tea, dairy products, vegetables, salad, fruits, and meat. The North and Northeast, with higher DII values, had a significantly higher intake of flour, pasta, and starch. All of these findings related to regional differences may be explained by the history of colonization (Italy and German in the South and Southeast and Portugal in the Northeast, for instance) and lifestyle in this huge continental country [43].

\section{Limitations and perspectives}

Our study has some limitations, such as a lack of measurements of CRP serum levels or other inflammatory biomarkers for comparison with the DII. However, previous studies regarding the DII have shown its ability to estimate CRP levels in other populations. Furthermore, we did not perform any spine radiograph to evaluate morphometric vertebral fractures or DXA measurements for diagnosis of osteoporosis. Also, its cross-sectional design and use of one 24-h recall interview pose another limitation. Another limitation of the 24-h recall is that only 24 out of the 45 food parameters were available for calculating DII scores. It is likely that the results would have been better with more food parameters, such as flavonoids, ginger, onions, and garlic. Further studies with larger sampling size and longitudinal design, particularly intervention clinical trials, are needed to establish the association between the DII and low-impact fractures.

\section{Conclusions}

In Brazil, food consumption is basically represented by energy-dense, nutrient-sparse foods, contributing to a higher pro-inflammatory potential. In the present study, women had a higher DII compared to men. We did not find any associations with higher DII and low impact fractures. The profile of women taking statins in this study was associated with diet pattern that were potentially anti-inflammatory. Additionally, because of the cross-sectional design of this study, we are not able to establish a causal relationship. 


\section{Acknowledgements}

Dr. Hébert was supported by an Established Investigator Award in Cancer Prevention and Control from Cancer Training Branch of the National Cancer Institute (K05 CA136975). Dr. Pinheiro was funded by a grant from Wyeth Consumer Healthcare.

\section{Funding}

Our study was supported by an Established Investigator Award in Cancer Prevention and Control from Cancer Training Branch of the National Cancer Institute (K05 CA136975) and funded by a grant from Wyeth Consumer Healthcare.

\section{Availability of data and materials}

Database is available upon request.

\section{Authors' contributions}

MMP and LAM: were responsible for the study design, statistical analysis and the elaboration of the paper; NJS: performed all the nutrient calculation and participated in paper elaboration; PSG: participated in the nutrient calculation and conducted training about $24 \mathrm{~h}-\mathrm{R}$ for interviewers. NS: performed the Dietary Inflammatory Index calculation; MM: database review and nutrients reassessment for Dietary Inflammatory Index calculation, statistical analysis, results from analysis and elaboration of the paper. All authors read and approved the final manuscript.

\section{Ethics approval and consent to participate}

All participants gave written informed consent prior to participation in the study and the research ethics committee of the Universidade Federal de São Paulo/Escola Paulista de Medicina approved with the protocol number 1738/05.

\section{Consent for publication}

Not applicable.

\section{Competing interests}

Dr. James R. Hébert owns controlling interest in Connecting Health Innovations LLC (CHI), a company planning to license the right to his invention of the dietary inflammatory index (DII) from the University of South Carolina in order to develop computer and smartphone applications for patient counseling and dietary intervention in clinical settings. Dr. Nitin Shivappa is an employee of $\mathrm{CH}$. The subject matter of this paper will not have any direct bearing on that work, nor has that activity exerted any influence on this project. The authors have no other potential competing interest to disclose.

\section{Publisher's Note}

Springer Nature remains neutral with regard to jurisdictional claims in published maps and institutional affiliations.

\section{Author details}

${ }^{1}$ Rheumatology Division, Universidade Federal de São Paulo, Unifesp/EPM, Rua Leandro Dupre, 204, conjunto 74, São Paulo, Brazil. ${ }^{2}$ Cancer Prevention and Control Program, University of South Carolina, Columbia, SC 29208, USA. ${ }^{3}$ Department of Epidemiology and Biostatistics, Arnold School of Public Health, University of South Carolina, Columbia, SC 29208, USA. ${ }^{4}$ Nutrition Division, Universidade do Vale do Paraiba, Univap, São José dos Campos, Brazil. ${ }^{5}$ Department of Nutrition, Faculdade de Saúde Pública, Universidade de São Paulo, São Paulo, Brazil.

Received: 15 October 2018 Accepted: 25 March 2019 Published online: 11 April 2019

\section{References}

1. Siris ES, Adler R, Bilezikian J, et al. The clinical diagnosis of osteoporosis: a position statement from the National Bone Health Alliance Working Group. Osteoporos Int. 2014. https://doi.org/10.1007/s00198-014-2655-z.

2. Pinheiro MM, Ciconelli RM, Martini LA, Ferraz MB. Clinical risk factors for osteoporotic fractures in Brazilian women and men: the Brazilian osteoporosis study (BRAZOS). Osteoporos Int. 2009. https://doi.org/10.1007/ s00198-008-0680-5.

3. Pinheiro MM, Neto ER, Machado FS, et al. Development and validation of a tool for identifying women with low bone mineral density and low-impact fractures: the São Paulo osteoporosis risk index (SAPORI). Osteoporos Int. 2012. https://doi.org/10.1007/s00198-011-1722-y.

4. Pinheiro MM, Reis Neto ET, Yang JHK, et al. Risk factors for osteoporotic fractures and low bone density in pre and postmenopausal women: the Sao Paulo osteoporosis study (SAPOS). Rev Saude Publica. 2010. https://doi. org/10.1590/S0034-89102010000300011.

5. Cavicchia PPSS, Hurley TG, Hussey JR, et al. A new dietary inflammatory index predicts interval changes in serum high-sensitivity C-reactive protein. J Nutr. 2009. https://doi.org/10.3945/jn.109.114025.

6. Ishii S, Cauley JA, Greendale GA, et al. C-reactive protein, bone strength, and nine-year fracture risk: data from the study of women's health across the nation (SWAN). J Bone Miner Res. 2013. https://doi.org/10.1002/jbmr.1915.

7. Yang S, Feskanich D, Willett WC, et al. Association between global biomarkers of oxidative stress and hip fracture in postmenopausal women: a prospective study. J Bone Miner Res. 2014. https://doi.org/10.1002/jbmr.2302.

8. Rivas A, Romero A, Mariscal-Arcas M, et al. Association between dietary antioxidant quality score (DAOs) and bone mineral density in Spanish women. Nutr Hosp. 2012. https://doi.org/10.3305/nh.2012.27.6.6039.

9. Prynne CJ, Mishra GD, O'Connell MA, et al. Fruit and vegetable intakes and bone mineral status: a cross-sectional study in 5 age and sex cohorts. Am J Clin Nutr. 2006. https://doi.org/10.1093/ajcn/83.6.1420.

10. Neale EP, Batterham MJ, Tapsell LC. Consumption of a healthy dietary pattern results in significant reductions in C-reactive protein levels in adults: a meta-analysis. Nutr Res. 2016. https://doi.org/10.1016/j.nutres. 2016.02.009.

11. Brighenti F, Valtueña S, Pellegrini N, et al. Total antioxidant capacity of the diet is inversely and independently related to plasma concentration of high-sensitivity C-reactive protein in adult Italian subjects. Br J Nutr. 2005. https://doi.org/10.1079/BJN20051400.

12. Bakker GC, van Erk MJ, Pellis $\mathrm{L}$, et al. An anti-inflammatory dietary mix modulates inflammation and oxidative and metabolic stress in overweight men: a nutrigenomics approach. Am J Clin Nutr. 2010. https://doi.org/10. 3945/ajcn.2009.28822.

13. Esposito K, Marfella R, Ciotola M, et al. Effect of a Mediterranean style diet on endothelial dysfunction and markers of vascular inflammation in the metabolic syndrome: a randomized trial. JAMA. 2004;292(12):1440-6. https:// doi.org/10.1001/jama.292.12.1440.

14. Bluher M, Rudich A, Kloting $N$, et al. Two patterns of Adipokine and other biomarker dynamics in a long-term weight loss intervention. Diabetes Care. 2012;35:342-9. https://doi.org/10.2337/dc11-1267.

15. Konstantinidou V, Covas M-I, Muñoz-Aguayo D, et al. In vivo nutrigenomic effects of virgin olive oil polyphenols within the frame of the Mediterranean diet: a randomized controlled trial. FASEB J. 2010;24:2546-57. https://doi. org/10.1096/fj.09-148452

16. Fung TT, Rimm EB, Spiegelman D, et al. Association between dietary patterns and plasma biomarkers of obesity and cardiovascular disease risk. Am J Clin Nutr. 2001:73:61-7. https://doi.org/10.1093/ajcn/73.1.61.

17. Shivappa N, Steck SE, Hurley TG, Hébert JR. Designing and developing a literature-derived, population-based dietary inflammatory index. Public Health Nutr. 2014;17:1689-96. https://doi.org/10.1017/S1368980013002115.

18. Shivappa N, Steck SE, Hurley TG, et al. A population-based dietary inflammatory index predicts levels of C-reactive protein in the seasonal variation of blood cholesterol study (SEASONS). Public Health Nutr. 2013;17: 1825-33. https://doi.org/10.1017/S1368980013002565.

19. Wirth M, Burch J, Shivappa N, et al. Association of a dietary inflammatory index with inflammatory indices and metabolic syndrome among police officers. J Occup Environ Med. 2014. https://doi.org/10.1097/JOM. 0000000000000213

20. Wood LG, Shivappa N, Berthon BS, et al. Dietary inflammatory index is related to asthma risk, lung function and systemic inflammation in asthma. Clin Exp Allergy. 2015;45(1):177-83. https://doi.org/10.1111/cea.12323.

21. Shivappa N, Hebert JR, Rietzschel, et al. Association between dietary inflammatory index and inflammatory markers in Asklepios study. Br J Nutr. 2015. https://doi.org/10.1017/S000711451400395X.

22. Shivappa N, Bosett C, Zucchett A, et al. Association between dietary inflammatory index and prostate cancer among Italian men. Br J Nutr. 2015: 113(2):278-83. https://doi.org/10.1017/S0007114514003572.

23. Orchard T, Yildiz V, Steck SE, et al. Dietary inflammatory index, bone mineral density, and risk of fracture in postmenopausal women: results from the women's health initiative. J Bone Miner Res. 2017:32:1136-46. https://doi. org/10.1002/jbmr.3070. 
24. Zhang ZQ, Cao WT, Shivappa N, et al. Association between diet inflammatory index and osteoporotic hip fracture in elderly Chinese population. J Am Med Dir Assoc. 2017. https://doi.org/10.1016/j.jamda.2017.02.011.

25. Veronese N, Stubbs B, Koyanagi A, Hébert JR, Cooper C, Caruso MG, Guglielmi G, Reginster JY, Rizzoli R, Maggi S, Shivappa N. Pro-inflammatory dietary pattern is associated with fractures in women: an eight-year longitudinal cohort study. Osteoporos Int. 2018;29:143. https://doi.org/10. 1007/s00198-017-4251-5.

26. Willett W, Stampfer MJ. Implications of total energy intake for epidemiological analyses. Am J Epidemiol. 1998;2:273-30. https://doi.org/10. 1093/oxfordjournals.aje.a114366.

27. Institute of Medicine Dietary Reference Intakes for Thiamin, Riboflavin, Niacin, Vitamin B6, Folate, Vitamin B12, Pantothenic Acid, Biotin, and Choline. Institute of medicine (US) standing committee on the scientific evaluation of dietary reference intakes and its panel on folate OBV, and choline. Washington: National Academies Press (US); 1998.

28. Institute of Medicine. Dietary reference intakes. Vitamin $\mathrm{C}$, vitamin $\mathrm{E}$ selenium and carotenoids. Washington, DC: National Academy Press; 2000.

29. Institute of Medicine. Vitamin a, vitamin K, arsenic, boron, chromium, copper, iodine, Iron, manganese, molybdenum, nickel, silicon, vanadium, and zinc. Washington, DC: National Academy Press; 2001.

30. World Health Organization. Physical status: the use and interpretation of anthropometry. Report of a WHO expert committee. WHO technical report series no. 854. Geneva: WHO; 1994.

31. Instituto Brasileiro de Geografia e Estatística. 2000. http://www.ibge.gov.br/ home/estatistica/populacao/censo2000/default.shtm. Accessed July 2016.

32. Shivappa N, Hébert JR, Karamati M, Shariati-Bafghi SE, Rashidkhani B. Increased inflammatory potential of diet is associated with bone mineral density among postmenopausal women in Iran. Eur J Nutr. 2016. https:// doi.org/10.1007/s00394-015-0875-4.

33. Artom N, Montecucco F, Dallegri F, et al. Carotid atherosclerotic plaque stenosis: the stabilizing role of statins. Eur J Clin Investig. 2014;55(2):561-8. https://doi.org/10.1007/s00394-015-0875-4.

34. Hu FB. Dietary pattern analysis: a new direction in nutritional epidemiology. Curr Opin Lipidol. 2002;13:3-9.

35. Barbaresko J, Koch M, Schulze MB, Nöthlings U. Dietary pattern analysis and biomarkers of low-grade inflammation: a systematic literature review. Nutr Rev. 2013;71:511-27. https://doi.org/10.1111/nure.12035.

36. Pinheiro MM, Ciconelli R, Chaves G, et al. Antioxidant intake among Brazilian adults - the Brazilian osteoporosis study (BRAZOS): a cross-sectional study. Nutr J. 2011;10:39. https://doi.org/10.1186/1475-2891-10-39.

37. Canela MD, Bastos DHM, Pinheiro MM, et al. Consumption of stimulant drinks and consequent ingestion of phenolic compounds and caffeine. Nutrire: rev Soc Bras Alim Nutr (J Brazilian Soc Food Nutr). 2009;34:143-57.

38. Pinheiro MM, Ciconelli RM, Martini LA, Ferraz MB. Risk factors for recurrent falls among Brazilian women and men: the Brazilian osteoporosis study (BRAZOS). Cad Saude Publica. 2010;26:89-96.

39. Campolina AG, Pinheiro MM, Ciconelli RM, Ferraz MB. Quality of life among the Brazilian adult population using the generic SF-8 questionnaire. Cad Saude Publica. 2011;27:1121-31.

40. Pereira RA, Duffey KJ, Sichieri R, Popkin BM. Sources of excessive saturated fat, trans fat and sugar consumption in Brazil: an analysis of the first Brazilian nationwide individual dietary survey. Public Health Nutr. 2014;17: 113-21. https://doi.org/10.1017/S1368980012004892.

41. Sichieri R, Castro JFG, Moura AS. Factors associated with dietary patterns in the urban Brazilian population. Cad Saude Publica. 2003;19:547-53.

42. Instituto Brasileiro de Geografia e Estatística. Household budget survey: analysis of food consumption in Brazil; 2011

43. Instituto Brasileiro de Geografia e Estatística. Household budget survey: food and beverage acquisition for household consumption per capita Brazil and major regions; 2011.

Ready to submit your research? Choose BMC and benefit from:

- fast, convenient online submission

- thorough peer review by experienced researchers in your field

- rapid publication on acceptance

- support for research data, including large and complex data types

- gold Open Access which fosters wider collaboration and increased citations

- maximum visibility for your research: over $100 \mathrm{M}$ website views per year

At $\mathrm{BMC}$, research is always in progress.

Learn more biomedcentral.com/submissions 